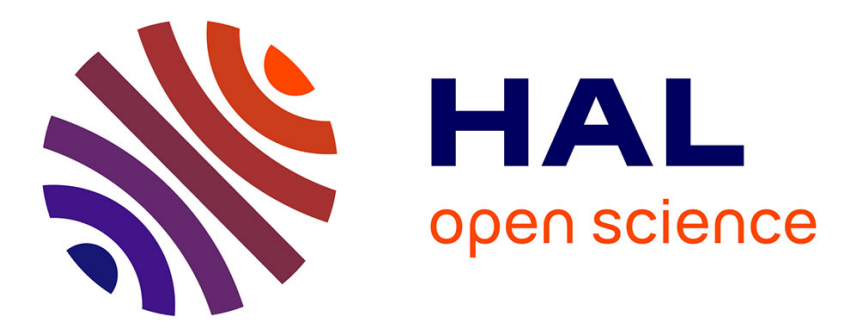

\title{
Une entreprise sidérurgique aux prises avec le groupware: apports d'une étude empirique
}

\author{
Valerie Nguyen Minh Lepine
}

\section{To cite this version:}

Valerie Nguyen Minh Lepine. Une entreprise sidérurgique aux prises avec le groupware: apports d'une étude empirique. Les Enjeux de l'information et de la communication, 2002, varia, 10.3917/enic.002.0065 . hal-01290743

HAL Id: hal-01290743

https://hal.univ-grenoble-alpes.fr/hal-01290743

Submitted on 17 Jul 2018

HAL is a multi-disciplinary open access archive for the deposit and dissemination of scientific research documents, whether they are published or not. The documents may come from teaching and research institutions in France or abroad, or from public or private research centers.
L'archive ouverte pluridisciplinaire HAL, est destinée au dépôt et à la diffusion de documents scientifiques de niveau recherche, publiés ou non, émanant des établissements d'enseignement et de recherche français ou étrangers, des laboratoires publics ou privés.

\section{(1) (1) $\$$}

Distributed under a Creative Commons Attribution - NonCommercial - NoDerivatives $\mid 4.0$ 


\section{Une entreprise sidérurgique aux prises avec le groupware : apports d'une éfude empirique}

Mise en ligne : 15 novembre 2002

Article inédit.

\section{Valérie Lépine}

Maître de conférences à l'Institut de la Communication de l'université Lumière Lyon 2, Valérie Lépine est responsable de la licence professionnelle «Communication Electronique ». Elle enseigne la sociologie et la communication des organisations. Ses centres d'intérêt : les relations entre évolutions des rapports sociaux et NTIC dans les organisations.

Plan

Introduction

De quelle réussite parle-t-on? La gestion des dossiers des essais métallurgiques Le suivi et la consolidation du bilan social

L'échec prévisible d'applications sans usages ou sans usagers

Qualigest : la gestion informatisée des documents et des procédures Qualité La gestion des demandes de travaux informatiques

Références bibliographiques

\section{INTRODUCTION}

Le travail qui consiste à observer, pour tenter de le comprendre et de l'expliquer, le phénomène objectif de diffusion des NTIC - parmi lesquelles le groupware - comme vecteur prééminent des dispositifs informationnels et communicationnels des organisations n'en est encore, en France en particulier, qu'à ses débuts. Aussi, les études empiriques décrivant l'introduction de groupware dans les organisations (Craipeau et Briole, 1995, 2000 ; Courbon et Tajan, 1997), qui rendent compte d'une réalité diverse et complexe, constituent-elles un matériau de départ utile à l'élaboration théorique de cette problématique dans le champ des sciences de la communication. Nous proposons, dans cet article, de présenter les résultats d'une étude qualitative conduite au siège parisien et au sein d'une des entités en province d'un important groupe de l'industrie sidérurgique.

Le groupe connaît en 1998 une profonde réorganisation de l'entreprise au niveau mondial ainsi qu'un vaste projet de refonte du système d'information (SI). Sur fond de restructurations, de fusions, de regroupements, de réduction des coûts et de suppression d'emplois, le groupe engagé dans la compétition internationale vise une stratégie de différenciation et d'efficience productive. L'entreprise souhaite passer d'une logique industrielle de production à une logique dite de service, orientée vers la satisfaction de ses clients. Pour les dirigeants, les NTIC et leur déploiement dans l'ensemble des multiples composantes du groupe sont un des pivots de cette orientation stratégique appuyée par la mise en œuvre de politiques sociales (Aménagement du Temps de Travail, démarche participative) et organisationnelles (Qualité Totale, sécurité, topo-maintenance, etc.). Au moment de l'enquête la grande réforme du SI est encore au stade de projet. Les applications informatiques développées depuis un an et demi sous le logiciel Lotus Notes par un responsable groupware recruté sur ce nouveau poste ne font que préfigurer le grand changement annoncé et d'une certaine manière constituent une phase de sensibilisation, de test préalable, dont il n'a pas été tiré de bilan officiel. Cette période de relative 
incertitude, où la marge de manœuvre des agents quant à la conception d'applications spécifiques, l'adoption ou non de nouvelles pratiques informationnelles et communicationnelles, est encore large, donne l'opportunité de saisir les logiques de l'évaluation intuitive qui émane des prescripteurs de ces techniques peu après l'introduction des NTIC dans l'organisation.

L'approche proposée pour saisir de telles logiques s'inscrit dans une problématique des communications organisationnelles qui vise à appréhender trois dimensions de la communication médiatisée par des dispositifs de type groupware:

- la dimension informationnelle qui concerne la gestion du flux des échanges et des activités de recherche et traitement de l'information ;

- la dimension relationnelle qui engage dans l'interaction professionnelle les régulations des rapports sociaux interindividuels et collectifs ;

- la dimension symbolique autour de laquelle se noue le sens donné à l'action autant que le langage et les représentations qui la légitiment.

À travers le cas présenté, nous voudrions montrer que ces dimensions, qui dépassent largement la simple question technique, sont étroitement imbriquées mais qu'elles doivent être distinguées car elles peuvent contribuer à une approche des évolutions des systèmes d'information sous un angle qui élargit le cadre instrumental qui constitue le plus souvent le seul référent des organisations.

L'étude concerne quatre applications développées sous Lotus Notes : deux sont décrites et vécues comme des expériences réussies ou satisfaisantes, tant du côté des commanditaires de l'application que des utilisateurs ; les deux autres, à l'inverse, s'avèrent ressenties comme des échecs, au moins du côté des utilisateurs.

\section{DE QUELLE RÉUSSITE PARLE-T-ON ?}

\section{La gestion des dossiers des essais métallurgiques}

L'entreprise sidérurgique étudiée produit des bobines d'acier laminé dont les caractéristiques sont définies en fonction des besoins spécifiques des clients. Le service des Essais est chargé de réaliser des essais techniques de matériaux qui peuvent être de toute nature (résistance, brillance, composition, etc.) et émaner de services divers (laboratoire de recherche, service des spécifications, service commercial principalement). Le chef du service a été l'initiateur d'une l'application de gestion informatisée de ces essais métallurgiques, en collaboration avec le responsable du groupware qui a assuré le développement de la maquette. Le processus commence avec la formulation initiale d'une demande et se termine par la constitution finale d'un rapport de résultats, en passant par la réalisation des essais à proprement parler. C'est un processus complexe impliquant de nombreux acteurs. Il n'est pas forcément linéaire, différents tests ou mesures pouvant être effectués en parallèle. Avant la mise en place d'une application de gestion de suivi de ce processus, l'ensemble des informations afférentes à un essai était rassemblé en un dossier papier centralisé par le service sans être accessible directement aux demandeurs.

L'idée de transposer ce dossier sur un support informatique est issue d'une double attente : la réduction de délais jugés beaucoup trop longs du côté des demandeurs, d'une part, la volonté d'alléger la charge de travail exprimée par les personnes réalisant les essais, compte tenu de l'accroissement permanent des demandes auxquelles elles doivent faire face, d'autre part: "Ils brassent des dossiers papier dans tous les sens (origine de la demande, fiche de lancement des essais, lotissements, notes techniques, rapports d'ateliers, etc.), ils sont surchargés de demandes (...) et nous sommes sans cesse interrompus par des 
questions ». De son côté le chef de service avait un intérêt personnel à alléger sa propre charge de travail, et notamment à réduire le nombre d'appels téléphoniques de personnes s'enquérant des résultats intermédiaires de leurs essais. Le service était aussi soumis à une démarche de certification pour l'obtention d'un label Qualité. Aussi la réflexion engagée répondait-elle à une volonté commune de remettre à plat l'ensemble des procédures du service, afin de les améliorer et de les stabiliser.

Après avoir dû renoncer à un premier projet trop coûteux, le chef de service s'est tourné vers le responsable du groupware. Une première rencontre a donné lieu à l'élaboration du cahier des charges, puis il n'aura fallu que deux jours pour qu'une première maquette Lotus Notes soit mise en place. C'est sur la base de cette version initiale que le chef de service a ensuite consulté une bonne partie des utilisateurs (méthodistes, laboratoire, etc.) qui seraient concernés par l'utilisation de l'application, ainsi que sa hiérarchie, pour approbation. Une seconde version, améliorée par la prise en compte des constats tirés des premiers dossiers informatisés, était en cours de test au moment des entretiens, et devait aboutir rapidement à la finalisation du projet.

Le bilan de cette application, tiré par le chef de service et par une personne utilisatrice du laboratoire, s'est avéré très favorable à la systématisation de son usage. Le premier point positif est lié à la méthode de déploiement procédant par essais, consultations des utilisateurs et ajustements successifs : il a permis de ne pas passer à côté d'oppositions ou de réticences particulières à l'égard de l'outil technique. En second lieu, l'application semble répondre aux besoins exprimés en amont de son développement, puisque les demandeurs d'essais peuvent désormais accéder aux résultats intermédiaires des tests, directement et sans solliciter la bienveillance d'un membre du service. Le travail aurait donc gagné à la fois en qualité et en rapidité dans l'exécution de ses missions : "Il y a un gain de temps important! Les gens sont renseignés même quand je suis absent, et je lance plus d'essais (...), je me consacre à la qualité des rapports, ça permet de faire plus de recherche, d'organisation, d'être plus précis dans les comptes-rendus ».

Les bénéfices apportés sont donc perçus comme réels et utiles pour les uns comme pour les autres, et l'application a été jugée par la Direction Industrielle comme suffisamment performante - grâce aux gains de productivité effectivement obtenus - pour servir d'exemple à d'autres sites de production. Il s'agirait là d'une «sucess story » où tous les facteurs de la réussite auraient été par avance réunis.

Le questionnement portant sur la portée communicationnelle de tels dispositifs, il est utile d'aller au-delà de ce premier bilan qui rend compte de la dimension informationnelle du projet pour examiner la portée de cette application, sur d'autres dimensions.

Tout d'abord, il apparaît que toute une partie de la population concernée par l'application d'informatisation du dossier essais, a été exclue de la consultation : les ouvriers de production, qui réalisent une partie des essais sur machines en atelier, en plus de leur tâche de production des produits standards, ont été tenus à l'écart.

L'étude révèle que les ouvriers sont, par principe, exclus du processus global de diffusion de l'outil informatique en cours dans l'ensemble du groupe : «de toute façon, leur patron la Direction de la production - ne veut pas qu'ils perdent du temps sur l'informatique». Cette politique de "mise à l'écart » qui se prolonge dans la période dite de modernisation des entreprises (Linhart, 1994) n'est pas totalement nouvelle, si l'on considère l'histoire des conflits qui ont, depuis des décennies, opposé ouvriers et patronat dans le monde de la sidérurgie : "L'identité d'Usinor a aussi été façonnée par une communication construite dans la confrontation et la revendication » (Godelier, 1999). 
Par ailleurs, lorsqu'ils ont - à cause des tâches qui leur sont demandées - affaire à l'informatique, les ouvriers sont dans une situation objective de domination. C'est le cas concernant l'outil de suivi des essais avec lequel on leur demande désormais de rédiger leurs comptes-rendus. Cette domination s'exerce essentiellement sur un plan symbolique, c'est-à-dire, ici, à travers l'imposition d'un langage légitime imposé par un service à travers l'outil technique : «Eux, les ouvriers, ils subissent plus qu'ils n'agissent. Nous, on veut que notre base soit propre, donc on veut avoir une information stéréotypée. On ne veut pas que n'importe qui arrive avec ses propres mots. On a mis des règles en place ».

Les règles langagières imposées le sont tant au niveau du contenu qui doit être restreint à l'essentiel, qu'au niveau de la forme (exemple : le nom de l'outil utilisé tout en majuscules, le nom de la personne qui a réalisé l'essai avec seule la première lettre en majuscule). Il y a là une illustration du travail de codification et de normalisation à l'œuvre dans les démarches qualité de standardisation des procédures (Mispelblom, 1999 ; Courpasson, 1996), auxquelles l'informatisation est souvent associée. L'imposition d'un glossaire ignorant les langages professionnels ouvriers constitue une contrainte qui contraste fortement avec le choix du chef de service, de sélectionner dans le logiciel les fonctionnalités qui lui permettent une expression peu formelle et libre pour la rédaction de ses propres rapports : «Il n’y a pas de champs pré-formatés : avec le système des pièces jointes, je peux m'éclater sur un dossier si je veux!».

Une dernière critique peut être adressée à cette application : elle est liée au pouvoir conféré par la maîtrise de la gestion des droits d'accès. En effet, l'attribution des droits d'accès à la base Notes ainsi que la définition de la nature de ces droits (consultation uniquement, droit d'écriture, droit de modification ou de suppression de documents), est exercée de façon totalement discrétionnaire par le titulaire de l'application : "Comme c'est moi le propriétaire de l'application, c'est moi qui décide, j'ai fait ma dictature pour les droits d'accès! ». Cette prérogative confère à ce responsable une autorité et une légitimité qui s'étendent à des entités sur lesquelles il n'a en principe aucune autorité hiérarchique, puisqu'il peut décider souverainement que dans tel service demandeur, telle personne a (ou n'a pas) le droit de consulter la base d'informations.

On voit ici qu'une application considérée comme exemplaire sur le plan de son efficience informationnelle pour une catégorie de personnel peut témoigner (voire renforcer) de profonds déséquilibres des relations de travail entre «cols blancs » et ouvriers. Ces derniers demeurent écartés des phases de conception de l'application et, plus gravement, ignorés dans le processus symbolique de désignation du langage et des codes fixés dans et par le dispositif technique.

\section{Le suivi et la consolidation du bilan social}

Dans ce groupe de 50000 personnes la gestion des ressources humaines est particulièrement complexe car le personnel est issu des recompositions par fusion ou rachat de sociétés aux conventions toutes différentes. C'est pourquoi la question de l'uniformisation des outils de GRH se pose avec une grande acuité. Les pratiques locales sont fortement ancrées et diffèrent d'un site à l'autre car chaque Directeur des ressources humaines jouit d'une grande autonomie tant pour la gestion des effectifs du site que pour la négociation salariale. Cette autonomie semble d'autant plus difficile à remettre en question que les DRH du groupe se trouvent être assez souvent d'anciens militaires de carrière, replacés à ces postes par l'État, et très attachés à leurs prérogatives. Aussi n'est-il pas étonnant que l'une des méthodes d'homogénéisation des pratiques - au moins de suivi, sinon de gestion sociale - ait été celle de la mise en place d'une informatique commune à l'ensemble des entités du groupe. Le système a subi plusieurs remaniements au cours des 
dix dernières années mais il est à nouveau refondu en 1997 à l'occasion de l'arrivée du responsable groupware, en réponse à la nouvelle législation qui impose à toutes les entreprises d'une certaine taille la collecte de données spécifiques pour l'établissement du bilan social : «Le plus gros est sur Excel, c'est une application très assistée. (...) En 1997, Mr T. est apparu et il a fait tout ça propre en mettant toutes nos applications sous Notes : il y avait la problématique des droits d'accès que les DRH de chaque site voulaient maîtriser».

Comme pour la précédente application étudiée, le projet a fait l'objet d'une assez large consultation par le siège auprès des utilisateurs finaux des différents sites. Cependant, dans ce cas-ci, ce n'est pas la maquette de développement qui a été discutée mais, à l'inverse de la précédente, le contenu, le sens accordé aux codes retenus : «La maquette est imposée mais, au niveau du contenu de chaque indicateur, on a travaillé ensemble ».

Du point de vue des utilisateurs locaux, la méthode consistant à imposer l'outil technique n'a pas nécessairement été bien vécue, d'autant que la formation à l'utilisation de l'outil a été quasiment inexistante ( On a eu un mémo pour dire maintenant, c'est Lotus Notes, on se plie aux exigences de la holding, nous ne sommes que des exécutants. ») ; elle n'a pas non plus surpris les employés des RH, habitués à voir le système de saisie changer régulièrement par décision unilatérale. La réussite de cette nouvelle application tient, du point de vue du commanditaire, au siège, à ses fonctionnalités techniques : facilité et rapidité de traitement et de consolidation de données devenues homogènes; rapidité de la mise à jour électronique en ligne en remplacement de l'ancien système qui procédait par échanges de disquettes. Mais le succès de ce dispositif est d'une autre nature du point de vue des utilisateurs sur site.

En effet, ce que souligne l'employé interviewé sur cette application, c'est précisément la satisfaction d'avoir été étroitement impliqué dans le travail de définition et de sélection des indicateurs pertinents pour l'établissement du bilan social. Est aussi particulièrement appréciée l'occasion, créée par cette circonstance, de rencontrer, pour la première fois, des collègues effectuant le même travail sur des sites géographiquement dispersés : "Pour uniformiser les rubriques, les codes de paye, on a fait des réunions de travail. C'est très enrichissant de rencontrer des gens qui font la même chose que vous, on visite les ateliers, on sort de notre contexte ». C'est donc le bénéfice communicationnel et relationnel qui est mis en avant, et qui, d'une certaine manière crée ou du moins favorise les conditions d'acceptabilité du résultat applicatif.

On peut conclure de la comparaison de ces deux applications, présentées par leurs responsables comme des réussites, qu'elles diffèrent essentiellement par la méthode de conception et d'implication des personnels. L'une a privilégié la pertinence du procès et de la forme technique de l'échange tandis que l'autre a privilégié la recherche d'un consensus concerté sur le contenu informationnel et sur le sens commun accordé aux codes de communication.

Il y a dans les deux cas uniformisation des procédures et des langages par le biais de l'outil de traitement de l'information; mais, là où la première application tend à faire violence à des comportements constitutifs de l'échange (la manière de s'exprimer par écrit, par exemple), l'autre laisse une marge de manœuvre et d'interprétation aux personnes. Par exemple, s'il est impératif de signaler, dans le bilan social, le nombre d'incidents liés à la sécurité, il n'y a pas de codification absolue pour décider de ce qui est, ou de ce qui n'est pas, jugé comme un incident : "Avoir le même outil, ça les aide à communiquer entre eux, ils parlent tous avec le même logiciel. Mais ça n'a pas forcément changé les comportements. Pour compter les incidents, chacun fait comme il veut ». 
Certes, cette marge de manœuvre, laissée au libre arbitre des personnes, peut affecter la précision des indicateurs de sécurité mais, en laissant place à une certaine autonomie de décision, elle rend aussi possible, à travers les échanges de face-à-face et les décisions prises en commun, une évolution des individus vers la pleine conscience des ajustements communicationnels qu'ils peuvent réaliser dans le cadre d'une collaboration véritable.

\section{L'ÉCHEC PRÉVISIBLE D'APPLICATIONS SANS USAGES OU SANS USAGERS}

\section{Qualigest : la gestion informatisée des documents et des procédures Qualité}

L'application est ici un progiciel développé sous Notes, acheté à une société spécialisée, et dédié à la gestion de documents Qualité. La décision d'utiliser le support informatique pour l'ensemble des documents a été prise deux ans avant cette investigation, par la direction de la Qualité au siège du groupe, dans le cadre d'une procédure de certification ISO 9002. Les procédures et documents du site en province avaient été formalisés sur papier depuis 1991 pour l'obtention de la certification ISO 9001. Il s'agissait donc simplement de remplacer les classeurs de documents papier par une application électronique, réputée faciliter la recherche d'information pour la rendre accessible à tous les services.

L'enquête a concerné des utilisateurs potentiels de cette base de données présentée comme l'application Notes la plus ancienne, censée concerner le plus grand nombre de salariés. En complément des entretiens une démonstration d'utilisation de Qualigest était demandée. Tout d'abord, de l'aveu général, la base Notes ayant été implantée par le siège sans aucune consultation locale, peu de responsables de services avaient pris la peine de s'y intéresser : "Les gens de l'assurance Qualité nous ont dit : ce sera comme ça. Il n’y a pas eu de véritable concertation, le risque, c'est qu'on n'utilise pas !». L'entretien a souvent constitué une première occasion de consulter la base de données, et les démonstrations ont été fort instructives. En effet, les obstacles techniques s'ajoutaient à des problèmes inhérents au contenu des bases et à la gestion des droits d'accès. Tout d'abord, sur le plan technique, il était nécessaire de disposer sur le poste de travail d'un logiciel spécifique (Acrobat Reader) qui permet de lire les pièces jointes au descriptif de procédure. Tous n'en disposaient pas et s'apercevaient de cette lacune en cours de démonstration. Ensuite, certains découvraient n'avoir que des droits d'accès restreints, et ne pouvaient consulter, par conséquent, que les procédures de leur propre entité alors qu'ils croyaient pouvoir disposer de l'ensemble des documents Qualité de l'entreprise. Pour finir, la base Qualigest semblait manifestement incomplète à ceux qui utilisaient habituellement les classeurs papier, en permanence à leur disposition : «Il manque un tas de procédures ! Et en plus je n'ai pas pu rentrer dans la base ». Ces problèmes révélés en séance n'avaient jamais été signalés au service central de la Qualité. Cela témoigne évidemment de la faible utilisation de l'outil informatique lorsqu'il est imposé, sans consultation ni implication des utilisateurs. Par ailleurs, Qualigest étant une application de mise à disposition de documents et d'informations, figés par le service Qualité et, par définition, stables dans la mesure où, précisément, la démarche vise à formaliser et à rendre reproductibles, à l'identique, des tâches selon un plan optimum, elle n'engage en rien une dimension coopérative particulière entre émetteurs des informations et utilisateurs de ce service.

Il s'agit au final d'une application "morte ", qui semble n'avoir d'autre utilité que de justifier, auprès des organismes certificateurs, l'informatisation d'un service informationnel comme une fin en soi. L'étude montre à quel point la constitution d'une base de données, fut-elle nécessaire, exhaustive et associée à un bon moteur de recherche, ne préjuge en rien de son inscription dans des usages. Classeur virtuel, cette application qui n’a jamais été 
remise en cause, est en réalité inopérante sur les trois dimensions informationnelle, relationnelle et symbolique.

\section{La gestion des demandes de travaux informatiques}

La seconde application très critiquée est celle qui permet d'effectuer sur son poste de travail des demandes qui concernent tous les travaux ou fournitures de matériels relevant du service informatique local du site de province. Ce type d'application professionnelle destinée aux services informatiques est courant dans les entreprises. Mis sur le marché précocement, de tels progiciels permettaient de convaincre les informaticiens des atouts de Lotus Notes, tout en mettant à leur disposition une application utile pour l'usage interne de leur service. Des entretiens ont été réalisés auprès du responsable du service exploitation en charge de traiter les demandes de travaux informatiques, ainsi qu'auprès de personnes de services émetteurs des requêtes.

Sur le principe, la démarche est assez similaire à celle rencontrée pour la gestion des essais métallurgiques, car il s'agit d'une application qui permet de faire électroniquement une demande de travaux par le biais d'un document pré-formaté. Puis cette demande est étudiée par le service compétent pour être évaluée en termes de faisabilité, de pertinence, jaugée en temps et ressources nécessaires, et chiffrée en coût. Après accord sur le budget et allocation des ressources disponibles, la tâche est attribuée à une personne en charge de la réalisation et de la livraison de la commande. Le logiciel doit aussi permettre au demandeur de suivre l'état d'avancement de son projet par consultation directe de la base Notes depuis le premier jour de sa requête jusqu'à la recette des travaux.

Là comme ailleurs, le processus existait déjà à l'identique, mais sous une forme plus traditionnelle. La demande était formulée par téléphone, accompagnée d'un dossier papier (formulaire de demande, cahier des charges et documents annexes) ou bien par messagerie avec des pièces jointes. Ce système ne suscitait pas de critiques importantes de la part des demandeurs et semblait assez opérationnel. Les plaintes émanaient en fait principalement du service informatique qui avait à gérer des demandes parvenant de partout, à tout moment et sous des formes extrêmement hétérogènes : « Les petites demandes, on risquait parfois de les perdre, et puis il y a toujours des demandes qui arrivent en catimini et qui perturbent tout le fonctionnement du service ».

C'est pour mieux contrôler et canaliser le flux des demandes de travaux - parfois vécues comme de véritables agressions - que le chef de service a décidé de mettre en place un outil de gestion informatisée. Il s'agit donc d'une application développée avec un véritable enjeu de gestion de la relation et pas seulement de meilleure maîtrise de l'information.

$\mathrm{Au}$ final, cette nouvelle application est jugée satisfaisante par le service informatique car elle autorise une meilleure visibilité de l'ensemble de la charge de travail et donne, par conséquent, de précieuses indications au responsable pour mieux gérer ses ressources humaines et ses priorités. Par ailleurs, le système rend plus explicite le budget correspondant à chaque demande, et permet d'évaluer de manière plus objective la rentabilité et, sur ce critère, la pertinence des travaux demandés. Le responsable interviewé estime qu'à terme cela aboutira à une diminution du nombre de demandes, dont il juge que certaines sont actuellement «inutiles» si elles ne sont pas rentables, sans autre évaluation plus qualitative. Du point de vue gestionnaire, l'application Notes a donc des avantages incontestables.

Le problème tient à ce qu'elle a été mise en place par et pour les informaticiens en charge de réaliser les travaux sans véritable écoute des attentes des demandeurs. La méthode de déploiement de l'application a d'ailleurs été brutale et directive tant dans sa forme que dans son contenu: "On a été autoritaires. C'est comme à la Sécu, il faut le bon 
formulaire !». Si les services les plus demandeurs ont été préalablement informés du projet, les autres - dont le nombre de demandes était inférieur à une ou deux par an - ont été totalement « ignorés ».

Contrairement à Qualigest qui peut être contournée grâce à la persistance des classeurs papier, l'application en question est devenue obligatoire pour voir une requête aboutir. De l'absence d'implication et de formation des utilisateurs, là encore, il résulte des formes d'utilisation aberrantes. C'est le cas de ce chef de service qui signe les formulaires électroniques des nouvelles demandes de travaux de son service encore vierges, car il ne parvient plus à effectuer cette manipulation de validation en fin de parcours, comme cela devrait être logiquement le cas.

Les remarques, les attentes formulées n'ont pas été prises en compte, ce qui témoigne du faible intérêt porté aux habitudes de travail et aux préoccupations des usagers au moment de la conception de l'application. Quelques exemples illustrent les critiques :

- les «champs» qui permettent de désigner un projet ne comportent que quelques caractères et ne permettent pas l'attribution de termes explicites ; la catégorisation des projets correspond à la préoccupation du seul service informatique (projet signé/à signer/en cours/soldé);

- en l'absence d'un moteur de recherche par mot-clef ou de "masque" créé par l'utilisateur pour permettre une consultation adaptée aux besoins de chacun, chaque catégorie doit être ouverte et consultée pas à pas pour retrouver la trace de la demande émise ;

- enfin, en l'absence de " pointeur » signalant les modifications ou les mises à jour correspondant à une demande, il est impossible de savoir si le projet a avancé sans consulter quotidiennement et exhaustivement la base.

Ces lacunes sont évidemment génératrices de perte de temps et d'agacement pour les usagers de ce service. Or, avec Lotus Notes, toutes ces fonctions sont accessibles, à condition d'y consacrer le temps de programmation nécessaire; leur défaut n'est par conséquent pas imputable aux limites techniques du logiciel, mais à l'ignorance des inconvénients pour l'utilisateur de cette application imposée sans concertation. Si l'application convient aux besoins gestionnaires du service qui l'a mise en place, elle est clairement insatisfaisante du point de vue de la qualité de la relation entre les agents, ainsi que du point de vue de la construction de significations et de codes communs nécessaires à l'intercompréhension dans la communication de travail.

\section{CONCLUSION}

Des travaux anglo-saxons appuyés sur des études empiriques de groupware (Ellis et alii, 1991 ; Orlikowski, 1992, 1997 ; Olson et Teasley, 1996) ont permis de recenser les freins ou les obstacles à la mise en place des outils de travail de groupe (Grudin, 1994) :

- surcroît de travail demandé par le passage à l'outil informatique ;

- difficulté à atteindre une masse critique d'utilisateurs (notamment dans le cas des forums ou groupes de discussion);

- ignorance des facteurs sociaux, politiques, des motivations réelles des acteurs ;

- rigidité des systèmes incapables de prendre en compte les exceptions ou anomalies qui ne manquent pas de se présenter ;

- ignorances des habitudes de travail et des usages antérieurs ; 
- incapacité d'évaluation réelle de ces dispositifs techniques dans le cadre complexe du travail de groupe ;

- décisions intuitives et non concertées des décideurs ;

- enfin, le plus souvent, absence des phases de test, d'ajustements, de formation préalablement à la mise en place du système définitif.

Notre étude conforte certains de ces points et plaide - s'il fallait encore le démontrer - en faveur d'une meilleure consultation des utilisateurs aux différentes étapes de conception et de déploiement des dispositifs de groupware.

Au-delà des aspects qui visent directement l'opérationnalité des démarches d'informatisation des systèmes informationnels, la recherche conduite en France pose, nous semble-t-il, un regard critique sur des dimensions moins souvent prises en compte dans les démarches anglo-saxonnes. En particulier il s'agit de ne pas négliger l'importance de la dimension symbolique du processus de normalisation-standardisation à l'œuvre dans les démarches d'informatisation comme celles liées à la Qualité (Cochoy et alii, 1998 ; Mispelblom, 1999). En analysant les contraintes de l'explicitation et de la formalisation des procédures de travail, ces auteurs insistent sur la production d'une écriture normative, d'un langage spécialisé qui viennent en contradiction avec les parlers ordinaires et les contrecatégorisations des opérateurs. Les dispositifs d'information et de communication étudiés ici s'inscrivent dans la même logique. En instituant avec force un langage et des modalités d'échange forgés par des acteurs en situation dominante, les dispositifs de groupware peuvent tendre à renforcer les dissymétries qui caractérisent la relation de travail héritée elle-même d'un rapport social historiquement ancré. Il serait illusoire ou erroné de ne considérer le succès ou l'échec d'une application que sous l'angle de son efficience informationnelle, sans se poser la question de sa contribution ou non à un effort de renouvellement de la relation et des communications de travail d'une part, de la coconstruction de significations et de représentations réellement partagées d'autre part. 


\section{RÉFÉRENCES BIBLIOGRAPHIQUES}

Briole Alain et Craipeau Sylvie, «Groupware et groupe : vers de nouveaux modes de coordination dans l'entreprise ? Hypothèses pour un cadre de recherche ", 2ème colloque de l'AIM : Vers des entrprises ouvertes?, Namur, mai 1995

Briole Alain et Craipeau Sylvie, «Le groupware. Une technique structurante pour les PME de service », Réseaux, vol. $18 \mathrm{n}^{\circ} 104,2000$

Cochoy Franck, Garel Jean-Pierre, De Terssac Gilbert, « Comment l'écrit travaille l'organisation : le cas des normes Iso 9000 », Revue Française de sociologie, n 39-4, 1998

Courbon Jean-Claude et Tajan Serge, Groupware et Intranet, application avec Notes et Domino, Inter Éditions, Paris 1997

Courpasson David, «Les normalisations managériales entre l'individu et le modèle professionnel », Revue d'économie industrielle, $\mathrm{n}^{\circ}$ 75, 1996

Ellis Clarence, Gibbs Simon et Rein Gail, « Groupware : some issues and experiences », Communications of the ACM, vol. 34/1, 1991

Godelier Éric, «Informer ou communiquer? Crise et communication chez Usinor », Gestion 2000. Actualités et perfectionnement, $\mathrm{n}^{\circ}$ 1, janvier 1999

Grudin Jonathan, «Groupware and social dynamics : eight challenges for developers », Association for Computing Machinery, Communications of the ACM, New York, janvier 1994 Lépine Valérie, Les enjeux communicationnels et socio-organisationnels du déploiement de groupware en entreprise, thèse de doctorat en Sciences de la communication, sous la direction de Bernard Miège, université Stendhal-Grenoble 3, décembre 2000

Linhart Danièle, La modernisation des entreprises, La Découverte, Paris, 1994

Mispelblom Fréderik, «Langages et stratégies au travail saisis par les normes d'assurance Qualité », Sociologie du travail, n $^{\circ}$ 3, vol. 4.1, 1999

Olson Judy et Teasley S. « Groupware in the wild : lessons learned from a year of virtual collocation », CSCW'96 Proceedings, Massachussets, 1996

Orlikowski Wanda, «Learning from Notes : organizational issues in groupware implementation ", Proceedings of the third conference on CSCW, Toronto, novembre 1992

Orlikowski Wanda et Hofman Debra, " An improvisional model of change management : the case of groupware technologies », Sloan Management Review, hiver 1997 